PROCEEDINGS OF THE

AMERICAN MATHEMATICAL SOCIETY

Volume 135, Number 11, November 2007, Pages 3405-3415

S 0002-9939(07)08673-X

Article electronically published on August 2, 2007

\title{
CODIMENSION GROWTH OF TWO-DIMENSIONAL NON-ASSOCIATIVE ALGEBRAS
}

\author{
A. GIAMBRUNO, S. MISHCHENKO, AND M. ZAICEV \\ (Communicated by Martin Lorenz)
}

\begin{abstract}
Let $F$ be a field of characteristic zero and let $A$ be a twodimensional non-associative algebra over $F$. We prove that the sequence $c_{n}(A), n=1,2, \ldots$, of codimensions of $A$ is either bounded by $n+1$ or grows exponentially as $2^{n}$. We also construct a family of two-dimensional algebras indexed by rational numbers with distinct T-ideals of polynomial identities and whose codimension sequence is $n+1, n \geq 2$.
\end{abstract}

\section{INTRODUCTION}

Let $A$ be a non-necessarily associative algebra over a field of characteristic zero. A natural and well-established way of measuring the polynomial identities satisfied by $A$ is through the study of the asymptotic behavior of its sequence of codimensions $c_{n}(A), n=1,2, \ldots$ More precisely, if $F\{X\}$ is the free (non-associative) algebra on a countable set $X=\left\{x_{1}, x_{2}, \ldots\right\}$ and $P_{n}$ is the space of multilinear polynomials in the variables $x_{1}, \ldots, x_{n}, c_{n}(A)$ is the dimension of $P_{n}$ modulo the polynomial identities satisfied by $A$.

It is well known that in case $A$ is an associative algebra satisfying a non-trivial polynomial identity (PI-algebra), the sequence of codimensions of $A$ is exponentially bounded ([12]). Also, in case $A$ is a Lie algebra, such a sequence can have overexponential growth ([11]). Nevertheless for both associative and Lie algebras, no intermediate growth (between polynomial and exponential) and no exponential growth between 1 and 2 is allowed $([8,[9,[10])$.

The class of algebras whose sequence of codimensions has exponential growth is quite wide. It includes all associative PI-algebras, a wide class of Lie algebras, all finite-dimensional algebras, etc. For any such algebra $A$ one defines the PI-exponent of $A$ as $\exp (A)=\lim _{n \rightarrow \infty} \sqrt[n]{c_{n}(A)}$, if it exists. It was proved in [4] and [5] that for any associative PI-algebra, the PI-exponent exists and is a non-negative integer (see also [6]). The same conclusion holds for any finite-dimensional Lie algebra ([14]). Nevertheless an example was constructed in [13] showing that Lie algebras

Received by the editors August 27, 2005 and, in revised form, February 9, 2006.

2000 Mathematics Subject Classification. Primary 17A50, 16R10; Secondary 16P90.

Key words and phrases. Polynomial identity, codimension growth.

The first author was partially supported by MIUR of Italy; the second author was partially supported by RFFI, grant 01-01-00739 and UR 04.01.036; the third author was partially supported by SSH, grant 1910.2003.1. 
exist whose sequence of codimensions grows exponentially but the rate of growth is not integer.

In the general case of non-associative algebras in [2] we constructed a family of algebras $A_{\alpha}$ whose sequence of codimensions has exponential growth, the exponent of $A_{\alpha}$ exists and equals $\alpha$, where $\alpha$ can be any real number greater than 1 .

Also, examples of non-associative algebras with intermediate growth of the codimensions can be constructed. For instance in 3, for any real number $0<\beta<1$, we constructed an algebra whose sequence of codimensions grows as $n^{n^{\beta}}$.

In 3 it was also shown that for any finite-dimensional algebra $A, \operatorname{dim} A=d$, $c_{n}(A)$ is either polynomially bounded or $c_{n}(A) \geq \frac{1}{n^{2}} 2^{\frac{n}{3 d^{3}}}$. On the other hand, for any $\beta>1, \varepsilon>0$, there exists a finite-dimensional algebra $B$ with $\beta-\varepsilon \leq \exp (B) \leq$ $\beta+\varepsilon([2])$.

In this paper we want to analyze in detail the codimension sequence of an arbitrary two-dimensional algebra. As in the case of associative or Lie algebras, it turns out that there is no intermediate growth and no exponential growth between 1 and 2. Hence the PI-exponent of any two-dimensional algebra is an integer. In fact we prove that the codimension growth of any two-dimensional algebra is either bounded by $n+1$ or is exponential with exponent equal to 2 . We also construct a family of two-dimensional algebras indexed by rational numbers with distinct T-ideals of polynomial identities and whose codimension sequence is $n+1, n \geq 2$.

This last result shows that the classification, up to PI-equivalence, of the twodimensional algebras is a difficult problem even in the case of linear codimension growth.

\section{Preliminaries}

Throughout this paper $F$ will be a field of characteristic zero and $A$ a nonnecessarily associative algebra. We let $X=\left\{x_{1}, x_{2}, \ldots\right\}$ be a countable set and $F\{X\}$ the free non-associative algebra on $X$ over $F$. For every $n \geq 1$, we consider $P_{n}$, the space of multilinear polynomials of $F\{X\}$ in the first $n$ variables $x_{1}, \ldots, x_{n}$. Note that $\operatorname{dim} P_{n}=C_{n} n$ !, where $C_{n}$ is the number of all possible arrangements of parentheses in a given monomial of length $n$. It is well known that $C_{n}=\frac{1}{n+1}\left(\begin{array}{c}2 n \\ n\end{array}\right)$ is the $n$-th Catalan number.

Given an algebra $A$, let $\operatorname{Id}(A)=\{f \in F\{X\} \mid f \equiv 0$ on $A\}$ be the T-ideal of $F\{X\}$ of polynomial identities of $A$. Since char $F=0$, it is well known that the sequence of spaces $P_{n} \cap \operatorname{Id}(A), n=1,2, \ldots$, carries all information about $\operatorname{Id}(A)$.

The symmetric group $S_{n}$ acts on the space $P_{n}$ by permuting variables: if $\sigma \in S_{n}$, $f\left(x_{1}, \ldots, x_{n}\right) \in P_{n}$,

$$
\sigma f\left(x_{1}, \ldots, x_{n}\right)=f\left(x_{\sigma(1)}, \ldots, x_{\sigma(n)}\right) .
$$

The space $P_{n} \cap \operatorname{Id}(A)$ is invariant under this action, and one studies the structure of $P_{n}(A)=P_{n} / P_{n} \cap \operatorname{Id}(A)$ as an $S_{n}$-module. The $S_{n}$-character of $P_{n}(A)$, denoted $\chi_{n}(A)$, is called the $n$-th cocharacter of $A$. Its degree $c_{n}(A)=\operatorname{dim} P_{n}(A)$ is the $n$-th codimension of $A$. By complete reducibility one writes

$$
\chi_{n}(A)=\sum_{\lambda \vdash n} m_{\lambda} \chi_{\lambda},
$$

where $\chi_{\lambda}$ is the irreducible $S_{n}$-character associated to the partition $\lambda$ of $n$ and $m_{\lambda} \geq 0$ is the corresponding multiplicity. 
Recall that if $\lambda \vdash n, d_{\lambda}=\chi_{\lambda}(1)$ denotes the degree of the irreducible character $\chi_{\lambda}$.

The next lemma provides a lower bound for the degree of a character corresponding to a rectangle of height two.

Lemma 2.1. Let $\lambda \vdash n$ be of the form $\lambda=(m, m)$ or $(m+1, m)$ or $(m+2, m)$ or $(m+3, m)$. Then if $n \geq 5$,

$$
d_{\lambda} \geq \frac{1}{n^{2}} 2^{n}
$$

Proof. By the hook formula ([7]) we have that for $k=0,1,2,3$,

$$
d_{(m+k, m)}=\frac{k+1}{m+k+1}\left(\begin{array}{c}
2 m+k \\
m
\end{array}\right) .
$$

Consider the well-known equality

$$
\sum_{i=0}^{n}\left(\begin{array}{l}
n \\
i
\end{array}\right)=2^{n}
$$

It is clear that in the sum on the left-hand side of (1) the greatest contribution is given by $\left(\begin{array}{c}n \\ m\end{array}\right)$ if $n=2 m$ or $n=2 m+1$ and by $\left(\begin{array}{c}n \\ m+1\end{array}\right)$ in the case $n=2 m+2$ or $n=2 m+3$. In any case such a term is greater than $\frac{2^{n}}{n+1}$.

Therefore, if $k=0$ or $k=1$ and $m \geq 2$,

$$
d_{(m+k, m)}>\frac{k+1}{(m+k+1)(n+1)} 2^{n}>\frac{1}{n^{2}} \cdot 2^{n} .
$$

Whereas if $k=2$ or $k=3$ and $m \geq 1$,

$$
\begin{aligned}
& d_{(m+k, m)}=\frac{(k+1)(m+1)}{(m+k)(m+k+1)}\left(\begin{array}{c}
n \\
m+1
\end{array}\right) \\
& >\frac{(k+1)(m+1)}{(m+k)(m+k+1)(n+1)} 2^{n}>\frac{1}{n^{2}} \cdot 2^{n} .
\end{aligned}
$$

The asymptotics of $d_{\lambda}$ for $\lambda=(m+k, m), k=0,1,2,3$, can be computed, and it turns out that $d_{(m+k, m)} \simeq \frac{4(k+1)}{n \sqrt{n}} 2^{n}$, where $n=2 m+k$. Hence the above lemma can be actually improved, and it can be shown that

$$
d_{\lambda} \geq \frac{C}{n \sqrt{n}} 2^{n}
$$

for some explicit constant $C>0$.

We next state the following result of Bahturin and Drensky that is the starting point for our investigation.

Lemma $2.2([1])$. Let $A$ be a finite-dimensional algebra, $\operatorname{dim} A=d$. Then $c_{n}(A) \leq$ $d^{n}$. 


\section{The MAin Result}

Throughout this section $A$ will be a two-dimensional algebra over a field $F$ of characteristic zero. We start by studying the case when $A$ contains a proper ideal with trivial multiplication.

Given a partition $\lambda$ of $n$ and a Young tableau $T_{\lambda}$ of shape $\lambda$, let $e_{T_{\lambda}}$ denote the essential idempotent corresponding to $T_{\lambda}$. Recall that $e_{T_{\lambda}}=\bar{R}_{T_{\lambda}} \bar{C}_{T_{\lambda}}$, where

$$
\bar{R}_{T_{\lambda}}=\sum_{\sigma \in R_{T_{\lambda}}} \sigma, \quad \bar{C}_{T_{\lambda}}=\sum_{\tau \in C_{T_{\lambda}}}(\operatorname{sgn} \tau) \tau,
$$

and $R_{T_{\lambda}}$ and $C_{T_{\lambda}}$ are the stabilizers of the rows and the columns of $T_{\lambda}$, respectively.

Lemma 3.1. Let $A$ be a two-dimensional algebra with a proper ideal $I$ such that $I^{2}=0$. Then

where $p \leq 2, q \leq 1$. Hence $c_{n}(A) \leq n+1$.

$$
\chi_{n}(A)=p \cdot \chi_{(n)}+q \cdot \chi_{(n-1,1)}
$$

Proof. Since $I$ is a proper ideal, $\operatorname{dim}_{F} I=1$, and let $\{a, b\}$ be a basis of the algebra $A$, with $a \in I$. Since $I^{2}=0$, then $a^{2}=(a b) a=a(b a)=0$.

Let

$$
\chi_{n}(A)=\sum_{\lambda \vdash n} m_{\lambda} \chi_{\lambda}
$$

be the decomposition of the $n$-th cocharacter of $A$ into irreducibles.

We first claim that $m_{\lambda}=0$ whenever $\lambda=\left(\lambda_{1}, \ldots, \lambda_{t}\right) \vdash n$ is such that $n-\lambda_{1}>1$.

In fact, let $T_{\lambda}$ be a Young tableau of shape $\lambda \vdash n$ with $\lambda_{3} \neq 0$. For any $f\left(x_{1}, \ldots, x_{n}\right) \in P_{n}$ such that $e_{T_{\lambda}} f\left(x_{1}, \ldots, x_{n}\right) \neq 0$, we have that $e_{T_{\lambda}} f=f_{1}+\cdots+$ $f_{m}$ with each polynomial $f_{i}$ alternating on at least three variables. Since $\operatorname{dim} A<3$ it follows that $e_{T_{\lambda}} f$ is an identity of $A$ and, so, $m_{\lambda}=0$ in this case. Similarly if $\lambda \vdash n$ is such that $\lambda_{2} \geq 2$, then for any tableau $T_{\lambda}$ and for any $f \in P_{n}$ with $e_{T_{\lambda}} f \neq 0$, the polynomial $e_{T_{\lambda}} f$ will be a sum of polynomials each alternating on two distinct sets of variables of order two. Also in this case $e_{T_{\lambda}} f \equiv 0$ on $A$ since $\{a, b\}$ is a basis of $A$ and $a^{2}=(a b) a=a(b a)=0$.

We have proved that if $m_{\lambda} \neq 0$ in (2), then $\lambda=(n)$ or $\lambda=(n-1,1)$.

Consider the partition $\lambda=(n-1,1)$ and let $M_{1}$ and $M_{2}$ be two irreducible $S_{n^{-}}$ submodules of $P_{n}$ corresponding to $\lambda$. It is well known that $M_{1}$ and $M_{2}$ are cyclic modules, and let $f_{1}\left(x_{1}, \ldots, x_{n}\right)$ and $f_{2}\left(x_{1}, \ldots, x_{n}\right)$ be generators of $M_{1}$ and $M_{2}$, respectively. Let $e_{T_{i}}$ be a Young tableau of shape $\lambda$ such that $e_{T_{i}} f_{i} \neq 0, i=1,2$. Since $e_{T_{i}}=\bar{R}_{T_{i}} \bar{C}_{T_{i}}$, it follows that $\bar{C}_{T_{i}} f_{i} \neq 0$. After multiplying on the left by suitable $\sigma, \tau \in S_{n}$, we may assume that $\sigma \bar{C}_{T_{1}} f_{1}$ and $\tau \bar{C}_{T_{2}} f_{2}$ are alternating on $x_{1}$ and $x_{2}$. Therefore, by replacing $\sigma \bar{C}_{T_{1}} f_{1}$ and $\tau \bar{C}_{T_{2}} f_{2}$ with $f_{1}$ and $f_{2}$, respectively, we may assume that $f_{1}$ and $f_{2}$ are alternating in the variables $x_{1}$ and $x_{2}$.

Since $I$ is an ideal of $A$ and $a \in I$, we have that $f_{i}(a, b, \ldots, b)=\alpha_{i} a$, for some $\alpha_{i} \in F, i=1,2$. We claim that $A$ satisfies the identity

$$
\alpha_{2} f_{1}-\alpha_{1} f_{2} \equiv 0 \text {. }
$$

In fact, if two or more variables are evaluated in $a$, we will get 0 since $I^{2}=0$. If we substitute $x_{1}=x_{2}=b$ we will also get 0 since $f_{1}$ and $f_{2}$ are alternating on $x_{1}$ and $x_{2}$. If we substitute $x_{1}=a, x_{2}=b$, we will get 0 by the definition of the scalars $\alpha_{1}, \alpha_{2}$. Finally, if $x_{1}=b$ and $x_{2}=a$, we will get 0 since $f_{i}$ is alternating on $x_{1}$ and $x_{2}$ and, so, $f_{i}(a, b, \ldots)=-f_{i}(b, a, \ldots)$. This establishes the claim. Thus $M_{1}=M_{2}$ 
modulo $P_{n} \cap \operatorname{Id}(A)$, and since both modules correspond to the partition $(n-1,1)$, we obtain that $m_{(n-1,1)} \leq 1$.

We next consider the partition $\lambda=(n)$.

Let $f_{i}, i=1,2,3$, be multilinear polynomials generating three submodules of $P_{n}$ corresponding to $\lambda=(n)$. In this case all variables of $f_{i}\left(x_{1}, \ldots, x_{n}\right)$ are symmetric for $i=1,2,3$.

We split the proof into two cases according to the multiplication table of $A$.

Case 1. Suppose that $a b=\alpha a, b a=\beta a$ and $b^{2}=\gamma b$, for some $\alpha, \beta, \gamma \in F$. Let $f_{i}(a, b, \ldots, b)=\alpha_{i} a$ and $f_{i}(b, \ldots, b)=\beta_{i} b, i=1,2,3$.

We claim that the algebra $A$ satisfies the identity

$$
\delta_{1} f_{1}+\delta_{2} f_{2}+\delta_{3} f_{3} \equiv 0,
$$

where $\left(\delta_{1}, \delta_{2}, \delta_{3}\right)$ is a solution of the following system of linear equations in the variables $z_{1}, z_{2}, z_{3}$ :

$$
\begin{gathered}
\alpha_{1} z_{1}+\alpha_{2} z_{2}+\alpha_{3} z_{3}=0 \\
\beta_{1} z_{1}+\beta_{2} z_{2}+\beta_{3} z_{3}=0 .
\end{gathered}
$$

In fact, since the $f_{i}$ s are symmetric on all variables and $I^{2}=0$, only two essentially different substitutions are possible: $x_{1}=\cdots=x_{n}=b$ and $x_{1}=a, x_{2}=\cdots=$ $x_{n}=b$. It is clear that the polynomial in (3) will vanish on these substitutions by the definition of the scalars $\delta_{1}, \delta_{2}, \delta_{3}$. Hence $m_{(n)} \leq 2$, and we are done in this case.

Case 2. Suppose that $a b=\alpha a, b a=\beta a$ and $b^{2}=\delta a+\gamma b$, for some $\alpha, \beta, \gamma, \delta \in F$, with $\delta \neq 0$. We note that by choosing the basis $\{\delta a, b\}$ we may assume that $a^{2}=0$, $a b=\alpha a, b a=\beta a$ and $b^{2}=a+\gamma b$.

Set $b^{\prime}=\varepsilon a+b$ for some $\varepsilon \in F$ and compute $\left(b^{\prime}\right)^{2}=(\varepsilon a+b)(\varepsilon a+b)=$ $(\varepsilon \alpha+\varepsilon \beta+1-\varepsilon \gamma) a+\gamma(\varepsilon a+b)$. If $\gamma \neq \alpha+\beta$, then we can set $\varepsilon=\frac{1}{\gamma-(\alpha+\beta)}$ and $\left\{a, b^{\prime}\right\}$ is a basis of $A$ satisfying the hypothesis of Case 1 .

Therefore we may assume that $\gamma=\alpha+\beta$, and we have that

$$
a^{2}=0, a b=\alpha a, b a=\beta a \text { and } b^{2}=a+(\alpha+\beta) b .
$$

Note that in this case the basis $\{a, a+b\}$ has the same multiplication table as $\{a, b\}$.

Let $f_{i}(b, \ldots, b)=\alpha_{i} a+\beta_{i} b, i=1,2,3$, and let $\left(\delta_{1}, \delta_{2}, \delta_{3}\right)$ be a solution of the system

$$
\begin{gathered}
\alpha_{1} z_{1}+\alpha_{2} z_{2}+\alpha_{3} z_{3}=0 \\
\beta_{1} z_{1}+\beta_{2} z_{2}+\beta_{3} z_{3}=0 .
\end{gathered}
$$

We claim that the algebra $A$ satisfies the identity

$$
\delta_{1} f_{1}+\delta_{2} f_{2}+\delta_{3} f_{3} \equiv 0 .
$$

In fact, if we substitute $x_{1}=\cdots=x_{n}=b$ or $x_{1}=\cdots=x_{n}=a+b$, we will get 0 . On the other hand, if we set $x_{1}=a$ and $x_{i}=b, i=2, \ldots, n$, we will also get 0 since by the symmetry of $f_{i}$ on all variables we have

$$
f_{i}(a, b, \ldots, b)=\frac{f_{i}(a+b, \ldots, a+b)}{n}-f_{i}(b, \ldots, b) .
$$

This proves the claim and $m_{(n)} \leq 2$ also in this case. Therefore $m_{(n)} \leq 2$ and the lemma is proved. Since by the hook formula, $\chi_{(n)}(1)=d_{(n)}=1$ and $\chi_{(n-1,1)}(1)=$ $d_{(n-1,1)}=n-1, c_{n}(A) \leq n+1$. 
In order to simplify the notation, in what follows we shall substitute alternation on a given set of variables in a polynomial with the marking of the variables with the same symbol $\left(^{-}, \sim\right.$, etc.). For instance, the polynomial

$$
\sum_{\sigma, \tau \in S_{2}}(\operatorname{sgn} \sigma \tau)\left(\left(\left(x_{\sigma(1)} x_{\tau(1)}\right) x_{\sigma(2)}\right) x_{\tau(2)}\right)
$$

will be simply written as $\left(\left(\left(\bar{x}_{1} \tilde{x}_{1}\right) \bar{x}_{2}\right) \tilde{x}_{2}\right)$.

We now study the case when $A$ contains a proper ideal with non-trivial multiplication.

Lemma 3.2. Let $A$ be a two-dimensional algebra with a proper ideal $I$ such that $I^{2}=I$. Then either $c_{n}(A)=1$ or $\frac{1}{n^{2}} 2^{n} \leq c_{n}(A) \leq 2^{n}$.

Proof. Since $\operatorname{dim} A=2$, by Lemma 2.2, $c_{n}(A) \leq 2^{n}$. Hence we need only to show that for all $n \geq 1$, either $c_{n}(A)=1$ or $c_{n}(A) \geq \frac{1}{n^{2}} 2^{n}$.

Since $I$ is proper, $\operatorname{dim}_{F} I=1$ and let $\{a\}$ be a basis of $I$. Since $a^{2} \neq 0$, we may assume that $a^{2}=a$ and let $\{a, b\}$ be a basis of $A$. Then $b a=\alpha a$ for some $\alpha \in F$ and, if we set $b^{\prime}=-\alpha a+b$, it follows that $b^{\prime} a=0$. As a consequence, we may assume that the basis $\{a, b\}$ of $A$ is such that $a^{2}=a$ and $b a=0$.

We next split the proof in two distinct cases according to whether $a b=0$ or $a b \neq 0$.

Suppose first that $a b=0$. In case $b^{2}=\gamma b$ for some $\gamma \in F, b$ generates a 1dimensional ideal $J$. Hence $A=I \oplus J$ is a commutative associative algebra and $c_{n}(A)=1$. The lemma is proved in this case.

We may therefore assume that $b^{2}=\alpha a+\gamma b$, for some non-zero $\alpha \in F$.

First consider the polynomial

$$
g\left(x_{1}, x_{2}\right)=\sum_{\sigma, \tau \in S_{2}}(\operatorname{sgn} \sigma \tau)\left(\left(\left(x_{\sigma(2)} x_{\tau(2)}\right) x_{\sigma(1)}\right) x_{\tau(1)}\right)
$$

or in our other notation,

$$
g\left(x_{1}, x_{2}\right)=\left(\left(\left(\bar{x}_{2} \tilde{x}_{2}\right) \bar{x}_{1}\right) \tilde{x}_{1}\right)
$$

Then it is easily computed that $g(b, a)=\alpha a$.

We shall prove that $c_{n}(A) \geq \frac{1}{n^{2}} 2^{n}$. Since this inequality holds trivially for $n \leq 4$, we may assume that $n \geq 5$. Write $n=4 m+k$, with $0 \leq k \leq 3$ and consider the polynomial $g_{n}\left(x_{1}, x_{2}\right)$ which is the left-normed product of $m$ copies of the polynomial $g$ and $k$ copies of the variable $x_{1}$. In other words

$$
g_{n}\left(x_{1}, x_{2}\right)=\left(\cdots \left((\cdots(\underbrace{\left.g\left(x_{1}, x_{2}\right) g\left(x_{1}, x_{2}\right)\right) \cdots g\left(x_{1}, x_{2}\right)}_{m \text {-times }}) \underbrace{\left.x_{1}\right) \cdots x_{1}}_{k \text {-times }}) .\right.\right.
$$

It is readily checked that $g_{n}(b, a)=\alpha^{m+k} a \neq 0$. Moreover the complete linearization of $g_{n}\left(x_{1}, x_{2}\right)$ generates an irreducible $S_{n}$-submodule $M$ of $P_{n}$ corresponding to the partition $\lambda=(2 m+k, 2 m) \vdash n$ and $M \notin \operatorname{Id}(A)$. By Lemma 2.1 we obtain that $c_{n}(A) \geq \operatorname{dim} M=\chi_{\lambda}(1)=d_{\lambda} \geq \frac{1}{n^{2}} 2^{n}$.

Suppose now that $a b \neq 0$. Since $a$ forms a basis of $I$, then $a b=\beta a$ for some $\beta \in F, \beta \neq 0$ and, by replacing $b$ with $\beta^{-1} b$, we may assume that $a b=a$. Hence in this case

$$
a^{2}=a, b a=0, \text { and } a b=a
$$


If $n=2 m$ is even, we consider the commutator $\left[x_{1}, x_{2}\right]=x_{1} x_{2}-x_{2} x_{1}$ and the polynomial $h_{m}\left(x_{1}, x_{2}\right)$ obtained by taking the left-normed product of $m$ copies of $\left[x_{1}, x_{2}\right]$. Hence

$$
h_{m}\left(x_{1}, x_{2}\right)=\left(\cdots(\underbrace{\left.\left[x_{1}, x_{2}\right]\left[x_{1}, x_{2}\right]\right) \cdots\left[x_{1}, x_{2}\right]}_{m \text {-times }}),\right.
$$

and it is immediate to check that $h_{m}(a, b)=a \neq 0$. Let $M$ be the $S_{n}$-submodule of $P_{n}$ generated by the complete linearization of $h_{m}\left(x_{1}, x_{2}\right)$. Then $M$ is an irreducible module corresponding to the partition $\lambda=(m, m) \vdash n$ and by the above $M \notin \operatorname{Id}(A)$. But then by Lemma 2.1, $c_{n}(A) \geq \operatorname{dim} M=d_{\lambda} \geq \frac{1}{n^{2}} 2^{n}$, and we are done.

If $n=2 m+1$, then it is enough to consider the polynomial $h_{m}\left(x_{1}, x_{2}\right) x_{1}$ which still satisfies $h_{m}(a, b) a=a \neq 0$, and the conclusion follows as above.

The following is a well-known result of linear algebra. We prove it here for completeness.

Lemma 3.3. Let $\varphi$ and $\psi$ be linear transformations of a two-dimensional vector space $V$ over an algebraically closed field $F$. If $[\varphi, \psi]$ is a nilpotent transformation, then $\varphi$ and $\psi$ have a common eigenvector.

Proof. Fix a basis $\left\{a_{1}, a_{2}\right\}$ of $V$ on which the matrix of $\varphi$ has Jordan canonical form $A$ and let $B=\left(\begin{array}{ll}x & y \\ z & t\end{array}\right)$ be the matrix of $\psi$ in this basis.

Suppose first that $A=\left(\begin{array}{ll}\alpha & 0 \\ 0 & \beta\end{array}\right)$. If $\alpha=\beta$, then $A$ is a scalar matrix and any non-zero vector of $V$ is an eigenvector of $\varphi$. Hence we may assume that $\alpha \neq \beta$. By direct calculation

$$
[A, B]=\left(\begin{array}{cc}
0 & (\alpha-\beta) y \\
(\beta-\alpha) z & 0
\end{array}\right)
$$

and, since $[A, B]$ is nilpotent, we obtain that either $y=0$ or $z=0$. In the first case $a_{2}$ is a common eigenvector of $\varphi$ and $\psi$, and in the second case $a_{1}$ is such a common eigenvector.

Suppose now that $A$ is of the form $A=\left(\begin{array}{cc}\alpha & 1 \\ 0 & \alpha\end{array}\right)$. Then

$$
[A, B]=\left(\begin{array}{cc}
z & (t-x) \\
0 & z
\end{array}\right)
$$

and from nilpotency we obtain $z=0$. Hence $a_{1}$ is a common eigenvector of $\varphi$ and $\psi$, and we are done.

We are now in a position to prove the main result of this note.

Theorem 3.1. Let $A$ be a two-dimensional algebra over a field of characteristic zero. Then either $c_{n}(A) \leq n+1$ or $\frac{1}{n^{2}} 2^{n} \leq c_{n}(A) \leq 2^{n}$.

Proof. Since the codimensions of an algebra are not changed upon extension of the base field (see for instance [4]), we may assume that $A$ is an algebra over the algebraically closed field $F$.

For any $a \in A$ let $L_{a}$ and $R_{a}$ be the linear transformations of $A$ of left and right multiplication by $a$, respectively. Hence, for any $b \in A, L_{a}(b)=a b$ and $R_{a}(b)=b a$.

Suppose first that for any $a, b \in A$, the commutator $\left[L_{a}, L_{b}\right]$ is not nilpotent.

If $n=2 m+1$ is odd, we consider the element $v \in A$ such that $\left[L_{a}, L_{b}\right]^{m}(v) \neq 0$. We then construct the right-normed polynomial

$$
g_{m}\left(x_{1}, x_{2}, x_{3}\right)=\left(\tilde{x}_{1}\left(\tilde{x}_{2} \cdots\left(\bar{x}_{1}\left(\bar{x}_{2} x_{3}\right)\right) \ldots\right)\right)
$$


that we can also write in the form

$$
g_{m}\left(x_{1}, x_{2}, x_{3}\right)=\left[L_{x_{1}}, L_{x_{2}}\right]^{m}\left(x_{3}\right) .
$$

It is clear that $g_{m}(a, b, v)=\left[L_{a}, L_{b}\right]^{m}(v) \neq 0$. If we let $M$ to be the $S_{n}$-module generated by the complete linearization of $g_{m}\left(x_{1}, x_{2}, x_{3}\right)$, then $M$ is irreducible corresponding to the partition $(m+1, m)$ and, by the above, $M \notin \operatorname{Id}(A)$. By Lemma 2.2 and Lemma 2.1 we then obtain that $2^{n} \geq c_{n}(A) \geq d_{(m+1, m)} \geq \frac{1}{n^{2}} 2^{n}$.

Now let $n=2 m+2$ be even. Note that if there exists a non-zero element $c \in A$ such that $c d=d c=0$ for all $d \in A$, then $c$ generates a proper ideal of $A$ and we will be done by Lemma 3.1 and Lemma 3.2. Therefore, since $g_{m}(a, b, v) \neq 0$, we may assume that either $x_{4} g_{m}\left(x_{1}, x_{2}, x_{3}\right)$ or $g_{m}\left(x_{1}, x_{2}, x_{3}\right) x_{4}$ is not an identity of $A$. Let $N$ be the $S_{n}$-submodule of $P_{n}$ generated by the complete linearization of $x_{4} g_{m}\left(x_{1}, x_{2}, x_{3}\right)$ or $g_{m}\left(x_{1}, x_{2}, x_{3}\right) x_{4}$. It can be easily proved that for any $k$ dimensional algebra $A$ its cocharacter $\chi_{n}(A)$ lies in the strip of width $k$ for all $n=1,2, \ldots$. Hence the cocharacter of $A$ lies in a strip of height two, $N$ contains an irreducible submodule $M$ corresponding to the partition $(m+2, m)$ or $(m+1, m+1)$ and $M \notin \operatorname{Id}(A)$. As above we then obtain that $2^{n} \geq c_{n}(A) \geq \frac{1}{n^{2}} 2^{n}$.

In case the commutator $\left[R_{a}, R_{b}\right]$ is not nilpotent, for any $a, b \in A$, then the above argument can be applied in order to construct a left-normed polynomial that will lead to the desired conclusion.

Therefore we may assume that both commutators $\left[L_{a}, L_{b}\right]$ and $\left[R_{a}, R_{b}\right]$ are nilpotent, for all $a, b \in A$. We then apply Lemma 3.3, and we conclude that $A$ has a one-dimensional left ideal $F a$ and a one-dimensional right ideal $F b$.

If $F a=F b$, then $F a$ is a proper two-sided ideal of $A$, and by Lemma 3.1 and Lemma 3.2 the conclusion of the theorem follows.

Therefore we may assume that $F a \neq F b$. Clearly $\{a, b\}$ is a basis of $A$. Moreover $b a=0$ since $b a \in F a \cap F b=0$, and by eventually multiplying $a$ or $b$ by a suitable scalar we may assume that only the following possibilities occur: either $a^{2}=0$ or $a^{2}=a$ and either $b^{2}=0$ or $b^{2}=b$. We next examine the possible four cases.

Suppose first that $a^{2}=0$ and $b^{2}=0$. If $a b=0$, then $A$ has trivial multiplication and $c_{n}(A)=0$, for all $n>1$. If instead $a b \neq 0$, then $A^{2}=F a b$ is a one-dimensional ideal of $A$ and we are done by Lemma 3.1 and Lemma 3.2.

Suppose now that $a^{2}=a$ and $b^{2}=0$ and let $a b=\alpha a+\beta b$. If $\alpha=0$, then $F b$ is a one-dimensional ideal of $A$. If $\beta=0$, then $F a$ is a one-dimensional ideal of $A$. In any case we are done by Lemma 3.1 and Lemma 3.2. Therefore we may assume that $\alpha \neq 0$ and $\beta \neq 0$.

If $\alpha \neq 0$ and $n=2 m$, define the polynomial

$$
g_{m}\left(x_{1}, x_{2}\right)=\left(\overline{\bar{x}}_{1}\left(\cdots\left(\tilde{x}_{1}\left(\cdots\left(\left(\bar{x}_{1} \bar{x}_{2}\right) \tilde{x}_{2}\right) \cdots \overline{\bar{x}}_{2}\right) \cdots\right)\right)\right)
$$

obtained by first taking the left-normed product $y=\left(\left((x_{1} \underbrace{\left.\left.x_{2}\right) x_{2}\right) \cdots x_{2}}_{m \text {-times }})\right.\right.$, then the right-normed product $\left.\left.(\underbrace{x_{1} \cdots\left(x_{1}\left(x_{1}\right.\right.}_{(m-1) \text {-times }} y)\right)\right)$ and then by alternating the variables equidistant from the center. Note that

$$
g_{m}(a, b)=(a \cdots(a(((a b) b) \cdots b))) .
$$

Now, $(((a b) b) \cdots b)=\alpha^{m} a+\alpha^{m-1} \beta b$ and $\left(a \cdots\left(a\left(a\left(\alpha^{m} a+\alpha^{m-1} \beta b\right)\right)\right)\right)=\gamma a+$ $\alpha^{m-1} \beta^{m} b \neq 0$, for some $\gamma \in F$. Hence $g_{m}(a, b) \neq 0$, and the complete linearization 
of $g_{m}\left(x_{1}, x_{2}\right)$ generates an irreducible module not contained in $\operatorname{Id}(A)$. Clearly this module corresponds to the partition $\lambda=(m, m)$.

In case $n=2 m+1$ is odd, we only need to consider the polynomial $x_{1} g_{m}\left(x_{1}, x_{2}\right)$ obtained from (41) by adding the variable $x_{1}$. Also in this case we can construct an irreducible module corresponding to the partition $\lambda=(m+1, m)$ which is not contained in $\operatorname{Id}(A)$. But then, by Lemma 2.2 and Lemma 2.1 we get the desired conclusion of the theorem.

The case when $a^{2}=0$ and $b^{2}=b$ is clearly obtained from the above by exchanging the roles of $a$ and $b$.

We finally consider the case when $a^{2}=a$ and $b^{2}=b$ and let $a b=\alpha a+\beta b$. If $\alpha=0$ or $\beta=0$ we get that $F b$ or $F a$ is a one-dimensional ideal of $A$, respectively. Hence we are done by Lemma 3.1 and Lemma 3.2. Therefore we may assume that $\alpha \neq 0$ and $\beta \neq 0$. The operators of left and right multiplication by $a$ and $b$ in the basis $\{a, b\}$ are identified with the following matrices:

$$
L_{a}=\left(\begin{array}{cc}
1 & \alpha \\
0 & \beta
\end{array}\right), L_{b}=\left(\begin{array}{cc}
0 & 0 \\
0 & 1
\end{array}\right), R_{a}=\left(\begin{array}{ll}
1 & 0 \\
0 & 0
\end{array}\right), R_{b}=\left(\begin{array}{cc}
\alpha & 0 \\
\beta & 1
\end{array}\right) .
$$

Hence

$$
\left[L_{a}, L_{b}\right]=\left(\begin{array}{cc}
0 & \alpha \\
0 & 0
\end{array}\right),\left[R_{a}, R_{b}\right]=\left(\begin{array}{cc}
0 & 0 \\
-\beta & 0
\end{array}\right)
$$

and

$$
\left[R_{a}, R_{b}\right]\left(\left[L_{a}, L_{b}\right](b)\right)=-(\alpha \beta) b .
$$

For $n=4 m+1$ we construct the polynomial

$$
g_{m}\left(x_{1}, x_{2}, x_{3}\right)=\left(\left[R_{x_{1}}, R_{x_{2}}\right]\left[L_{x_{1}}, L_{x_{2}}\right]\right)^{m}\left(x_{3}\right) .
$$

In our notation it can be written as

$$
g_{m}\left(x_{1}, x_{2}, x_{3}\right)=\cdots\left(\left(\left(\bar{x}_{1}\left(\bar{x}_{2} x_{3}\right)\right) \tilde{x}_{2}\right) \tilde{x}_{1} \cdots .\right.
$$

Since $g_{m}(a, b, b)=(-\alpha \beta)^{m} b \neq 0$, the complete linearization of $g_{m}\left(x_{1}, x_{2}, x_{3}\right)$ generates an irreducible module not contained in $\operatorname{Id}(A)$. This module corresponds to the partition $\lambda=(2 m+1,2 m)$. In order to consider the other cases when $n=4 m+2,4 m+3,4 m+4$, we need only to multiply on the left the polynomial $g_{m}$ by $L_{x_{2}},\left[L_{x_{1}}, L_{x_{2}}\right], R_{x_{2}}\left[L_{x_{1}}, L_{x_{2}}\right]$, respectively. By Lemma 2.2 and Lemma 2.1 we obtain the desired conclusion, and the proof of the theorem is complete.

The purpose of the following proposition is twofold. We construct a family of twodimensional algebras $\left\{A_{\alpha}\right\}_{\alpha \in \mathbb{Q}}$ whose sequence of codimensions is $c_{n}\left(A_{\alpha}\right)=n+1$, $n \geq 1$. This will show in particular that in the previous theorem the inequality $c_{n}(A) \leq n+1$ cannot be improved. On the other hand we prove that any two distinct algebras of the family have distinct ideals of identities. This indicates that in the case of two-dimensional algebras, the sequence of cocharacters does not carry enough information of the corresponding T-ideals.

Let $\alpha$ be a rational number such that $\alpha>0$ and let $A_{\alpha}$ be the two-dimensional algebra with basis $\{a, b\}$ and multiplication table

$$
a^{2}=0, a b=a, b a=0, b^{2}=a+\alpha b .
$$

It is clear that $F a$ is a two-sided ideal of $A_{\alpha}$ with trivial multiplication.

Proposition 3.1. Let $A_{\alpha}$ be the two-dimensional algebra described above. Then $c_{n}\left(A_{\alpha}\right)=n+1$ and $\operatorname{Id}\left(A_{\alpha}\right) \neq \operatorname{Id}\left(A_{\beta}\right)$, if $\alpha \neq \beta$. 
Proof. By Lemma 3.1 the $n$-th cocharacter of $A_{\alpha}$ satisfies

$$
\chi_{n}(A)=p \cdot \chi_{(n)}+q \cdot \chi_{(n-1,1)},
$$

where $p \leq 2$ and $q \leq 1$. Hence $c_{n}\left(A_{\alpha}\right)=p \operatorname{deg} \chi_{(n)}+q \operatorname{deg} \chi_{(n-1,1)}=p+q(n-1)$, and, in order to prove the first statement of the proposition, we need to show that $p=2$ and $q=1$.

Consider the partition $\lambda=(n) \vdash n$ and the two polynomials of degree $n$ in the variable $x: l_{n}(x)=(\cdots((x x) x) \cdots x)$, the left-normed product of $x$ with itself $n$-times, and $r_{n}(x)=(x \cdots(x(x x)) \cdots)$, the right-normed product of $x$ with itself $n$-times. It is easily checked that

$$
l_{n}(b)=\left(1+\alpha+\alpha^{2}+\cdots+\alpha^{n-2}\right) a+\alpha^{n-1} b \text { and } r_{n}(b)=\alpha^{n-2} a+\alpha^{n-1} b .
$$

It follows that the algebra $A$ does not satisfy the identity $\gamma_{1} l_{n}(x)+\gamma_{2} r_{n}(x) \equiv 0$, for any scalars $\gamma_{1}, \gamma_{2} \in F$. It is clear that the linearizations of $l_{n}(x)$ and $r_{n}(x)$ generate two irreducible modules corresponding to the partition $(n)$, and they are linearly independent mod. $\operatorname{Id}(A)$. This proves that in (5), $p=2$.

Consider now the polynomial

$$
t_{n}\left(x_{1}, x_{2}\right)=\left(\cdots\left(\left(\bar{x}_{1} \bar{x}_{2}\right) x_{1}\right) \cdots x_{1}\right) .
$$

It is easily checked that $t_{n}(b, a)=-a \neq 0$. Also the complete linearization of $t_{n}\left(x_{1}, x_{2}\right)$ generates an irreducible $S_{n}$-module corresponding to the partition $(n-1,1)$, not contained in $\operatorname{Id}\left(A_{\alpha}\right)$. Hence in (5) we must have $q=1$, and the first part of the proposition is proved.

Now let $\alpha, \beta \in \mathbb{Q}$ and consider the polynomial

$$
f_{\alpha}\left(x_{1}, x_{2}, x_{3}\right)=\alpha \sum_{\sigma \in S_{2}}(-1)^{\sigma}\left(\left(x_{\sigma(1)} x_{\sigma(2)}\right) x_{3}\right)-\sum_{\sigma \in S_{2}}(-1)^{\sigma}\left(x_{\sigma(1)}\left(x_{3} x_{\sigma(2)}\right)\right) .
$$

By making the substitution $x_{1}=b, x_{2}=a$ and $x_{3}=b$ it is easily checked that $f_{\alpha}\left(x_{1}, x_{2}, x_{3}\right)$ is an identity of $A_{\beta}$ if and only if $\beta=\alpha$. Hence if $\alpha \neq \beta$ we have $\operatorname{Id}\left(A_{\alpha}\right) \neq \operatorname{Id}\left(A_{\beta}\right)$.

\section{REFERENCES}

[1] Y. Bahturin and V. Drensky, Graded polynomial identities of matrices, Linear Algebra Appl. 357 (2002), 15-34. MR1935223 (2003k:16034)

[2] A. Giambruno, S. Mishchenko and M. Zaicev, Codimensions of algebras and growth functions, (preprint) U. of Palermo, Dipartimento di Matematica ed Applicazioni, Preprint N. 274 (2005).

[3] A. Giambruno, S. Mishchenko and M. Zaicev, Algebras with intermediate growth of the codimensions, Adv. in Appl. Math. 37 (2006), 360-377. MR2261178 (2007g:16033)

[4] A. Giambruno and M. Zaicev, On codimension growth of finitely generated associative algebras, Adv. Math. 140 (1998), 145-155. MR.1658530 (99k:16049)

[5] A. Giambruno and M. Zaicev, Exponential codimension growth of P.I. algebras: an exact estimate, Adv. Math. 142 (1999), 221-243. MR.1680198(2000a:16048)

[6] A. Giambruno and M. Zaicev, Polynomial identities and asymptotic methods, AMS Mathematical Surveys and Monographs Vol. 122, Providence, R.I., 2005. MR.2176105

[7] G. James and A. Kerber, The representation theory of the symmetric group, Encyclopedia of Mathematics and its Applications, Vol. 16, Addison-Wesley, London, 1981. MR0644144 (83k:20003)

[8] A. Kemer, T-ideals with power growth of the codimensions are Specht (Russian), Sibirskii Matematicheskii Zhurnal 19 (1978), 54-69; translation in Siberian Math. J. 19 (1978), 37-48. MR 0466190 (57:6070)

[9] S. Mishchenko, On varieties of Lie algebras of intermediate growth, (Russian) Vest. Akad. Navuk BSSR Ser. Fiz.-Mat. Navuk 1987, no. 2, 42-45, 126. MR0898426 (88h:17031) 
[10] S. Mishchenko, Lower bounds on the dimensions of irreducible representations of symmetric groups and of the exponents of the exponential of varieties of Lie algebras (Russian), Mat. Sb. 187 (1996), 83-94; translation in Sb. Math. 187 (1996), 81-92. MR1380205(97d:17003)

[11] V. M. Petrogradsky, Growth of polynilpotent varieties of Lie algebras, and rapidly increasing entire functions (Russian), Mat. Sb. 188 (1997), no. 6, 119-138; translation in Sb. Math. 188 (1997), no. 6, 913-931 MR1479133(99a:17008)

[12] A. Regev, Existence of identities in $A \otimes B$, Israel J. Math. 11 (1972), 131-152. MR0314893 $(47: 3442)$

[13] M. V. Zaicev, S. P. Mishchenko, An example of a variety of Lie algebras with a fractional exponent, Algebra, 11. J. Math. Sci. (New York) 93 (1999), no. 6, 977-982. MR1698766 (2000f:17016)

[14] M. Zaicev, Integrality of exponents of growth of identities of finite-dimensional Lie algebras (Russian), Izv. Ross. Akad. Nauk Ser. Mat. 66 (2002), 23-48; translation in Izv. Math. 66 (2002), 463-487. MR1921808 (2003g:17004)

Dipartimento di Matematica e Applicazioni, Via Archirafi 34, 90123 Palermo, Italia

E-mail address: agiambr@unipa.it

Department of Algebra and Geometric Computations, Faculty of Mathematics and Mechanics, Ulyanovsk State University, Ulyanovsk 432700, Russia

E-mail address: mishchenkosp@.ulsu.ru

Department of Algebra, Faculty of Mathematics and Mechanics, Moscow State UniVERsity, Moscow, 119992 Russia

E-mail address: zaicev@mech.math.msu.su 